\title{
CONTEXT-AWARE WEBLOG TO ENHANCE COMMUNICATION AMONG PARTICIPANTS IN A CONFERENCE
}

\author{
Kosuke Numa, The Graduate University for Advanced Studies, Email: numa@grad.nii.ac.jp
}

Hideaki Takeda, National Institute of Informatics

Takuichi Nishimura, Yutaka Matsuo, Masahiro Hamasaki, Noriyuki Fujimura, Keisuke Ishida, Tom Hope, Yoshiyuki Nakamura, National Institute of Advanced Industrial Science and Technology

Satoshi Fujiyoshi, Kazuya Sakamoto, ALPHA SYSTEMS INC.

Hiroshi Nagata, Osamu Nakagawa, Eiji Shinbori, Dai Nippon Printing Co., Ltd.

Keywords: Academic conference support, Weblog, communication support.

Abstract: In this paper, we propose a Weblog system called ActionLog, which can associate Weblog entries to real world contexts. The real world context is not only useful for Weblog authors themselves, but also beneficial to communication among people, because people with the same or a similar context can easily find each other. ActionLog collects user actions from both Web-based and other real-world systems. Weblog contents are generated automatically from these user actions with related information. Users can browse and edit these entries as their personal action records and in addition can publish them for experience sharing. We built a practical system as an ActionLog application for conference support. Its main objective is to help people to learn about presentations and to effectively meet other people in a limited amount of time and the least amount of user commitment. There are three main features, i.e., integration of information in the real world and the cyber world, personalization in both information capturing from users and in information providing to users, and persistent assistance throughout the conference.

\section{INTRODUCTION}

Finding people with similar interests is an important activity in event spaces, such as academic conferences, conventions, and exhibitions. Thanks to quick diffusion of the Internet, our ability to obtain information and to know people is enhanced very much. We can meet people regardless of time and distance and we can obtain huge information instantly. On the other hand, our life becomes more complicated than one in the previous age. We should choose appropriate ways among various Internet applications depending on circumstances. Furthermore we do not live on the Internet all the time. Rather we still spend more time in the real world. So we should switch to realworld interaction from Internet interaction and vice versa frequently.

In this paper, we propose a Weblog system called ActionLog, which can associate Weblog entries to the real world context. The real world context is not only useful to Weblog authors, but also beneficial for communication among people because people with the same or similar contexts can easily find each other.

ActionLog is the concept for associating the real world context to the Weblog. We are now building two types of ActionLog applications. One is an application for open environments in which GPS-equipped mobile phones are used for capturing user context. The other is an application for conference support. We applied our system to two academic conferences. In this paper, we mainly report the system applied to UbiComp05, which is an academic conference for ubiquitous computing.

The paper is organized as follows. In the next section, we describe the concept of ActionLog. Then the sections following it will explain the ActionLog system for UbiComp05.

\section{BASIC IDEA}

Weblog has been widely spread all over the world. Many people use Weblog as a record of their dialy life like what they eat, whom they meet, what they buy and so on. Our system supports such authoring by adding context.

We model users' behaviors as actions with real world contexts. Action means what they do and the real world context is a set of information for the environment in which they do so. The real world context 
includes some physical situations like time and place, subjective situations like intentional objects, and social situations like people nearby.

The system manages to capture actions and contexts by using sensors and cooperating with other systems. Some contexts, like time and place can be obtained directly from sensors, but others should be inferred from sensor information. For example, we can obtain nearby people by integrating locational information of multiple users.

Then the system generates an entry for each action with sentences indicating the context of the action as a draft. Furthermore, the system shows related information, e.g., entries by other people with the same or similar contexts. This is achieved by integrating and calculating contexts.

The ActionLog usage scenario is as follows (Figure 1). The user walks around and does something with a wearable device like a mobile phone. They notify the device when they want to record an action. After they go back home, they browse with a PC a list of drafts, each of which corresponds to their actions, and edits and publishes some of them.

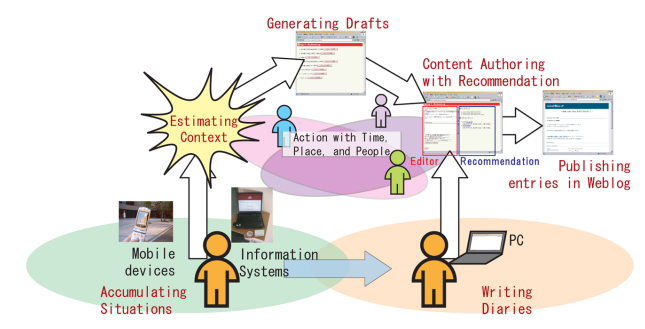

Figure 1: Usage scenario of the ActionLog system

\section{ACTIONLOG FOR CONFERENCES}

In the previous section, we explained the ActionLog in general. In this section, we focus on an ActionLog to support academic conferences.

\subsection{Supporting Academic Conferences}

Finding people with similar interests is an important activity in event spaces, such as academic conferences. Presenting information related to participants is helpful for this purpose. However, because of a time limitation and effort, it is almost impossible to look through all the information. It is also difficult to write and publish one's own information for sharing with other participants.
In this research, we help people with the idea of ActionLog. There are three features in the proposed way of assistance. One is that it offers integrated services between the real world and the cyber world. The system can gather and integrate users' information, like their actions both on site and on the web. Furthermore, users can access services either on site or from the Web. The second feature is that it is fully personalized. It does not simply mean that information can be selected according to users' profiles but that users can use the system as an assistant throughout the conference, i.e., they can settle a schedule, specify their acquaintances, and add their comments or journals with the system. The third feature is that it offers persistent services. Users can start to use the system before the conference and continue to use it after the conference without loosing information.

We built a conference support system called the Ubiquitous Community Support System (UbiCoSup) and ActionLog for a Conference (ActionLog-C) is one of the component systems of the UbiCoSup. Its main objective is to help people to learn about presentations and to effectively meet other people in a limited amount of time and with little user commitment. The basic functions of the UbiCoSup are: (1) visualizing researchers' social network, (2) presenting information about presentations and demonstrations, and (3) sharing experiences by using automatic Weblog generation from users' actions. The third one is realized by ActionLog. The system collects information by mining from the Web, by following users' instructions, and by capturing users' actions from systems. Then it integrates and presents the information.

\subsection{Action-oriented Experience Sharing Support}

The purpose of ActionLog-C is not only to support participants in composing their record of experiences but also to share experiences among participants. Sharing opinions or impressions among participants for an event serves an important role in characterizing the event and in communicating about it with other participants. We propose a way to share participants' experiences using ActionLog-C to achieve casual sharing of opinions among participants.

In this research, we assume an experience consists of an objectively observed action and her subjective thought at that time. To achieve experience sharing using information systems, we propose a method to capture user's actions, to generate contents from these actions, and sharing the contents among participants. Based on this method, the assistant agent helps the user to communicate with other participants. 


\subsection{Criteria to Design Communication}

To design communication by sharing action-based contents, the following points should be considered.

What kinds of actions are shared? We especially address event participants' interactions with information systems because they seem to be active and spontaneous actions for the system user. These interactions are efficiently collectable by the system and are easily understandable for participants.

When actions are shared? Meeting the other participants is an especially important activity in an event space. Action-based contents should be ready for sharing immediately after the actions occurred to support participants in finding participants with identical or similar interests. Previous works for sharing experiential contents do not process the experience in real time. PEPYS (Newman, 1991) and ComicDiary (Sumi, 2002) are also automatic content generation systems that generate diary-style contents dayby-day.

How actions are shared? It is important to reduce users' costs of capturing actions, creating contents, and sharing contents. Wearable computers can record all things the user sees and hears (Gemmell, 2002). Video and audio recordings help users to review their actions and share those experiences; nevertheless, using such large-scale equipment engenders high costs and is difficult to casually achieve. Sharing videos is also a difficult task for watchers of the contents. Textbased and/or other easily understandable expressions of experiences are needed.

With whom actions are shared? Sharing actions engenders privacy problems. As for location-based systems, some solutions, such as an anonymizing approach (Gruteser, 2003) or an obfuscation approach (Duckham, 2005), are proposed. However, in event spaces where participants wish to meet people, disclosure of the location and/or actions does not matter. It is sufficient for the user to select to publish or hide each action using a one-click operation.

\subsection{Basic Design for Experience Sharing}

To involve user's subjective thoughts to the contents, personal experience should be described by the user. For example, we cannot tell what Mr. A is doing when we find the situation where he is in a restaurant, eating some food, and talking about something with Ms. B. Mr. A might say he was having dinner, having an important discussion, or just dating with Ms. B. Therefore, we employ Weblogs as an infrastructure for contents sharing. People can easily express their thoughts or opinions with Weblog. In addition, Weblog offers an infrastructural aid to manage and share machine-readable contents (Karger, 2004).

The generated and published contents are aggregated according to their context. For example, extracting and listing the contents about a certain presentation achieves a topic-centric view (Avesani, 2005) on the presentation. However, to communicate with others, sharing contents is insufficient. Allowing users to reply to other contents can produce discussions.

The architecture of ActionLog-C is shown in Figure 2. The left-hand side of the figure indicates the action collection part of ActionLog-C. The system captures the user's actions from the other subsystems of the UbiCoSup. We describe this part in the next section. The center part indicates the core part of ActionLog-C, which can gather and accumulate information from other subsystems and offer it to users with different views. We explain the details of this process in Section 5.

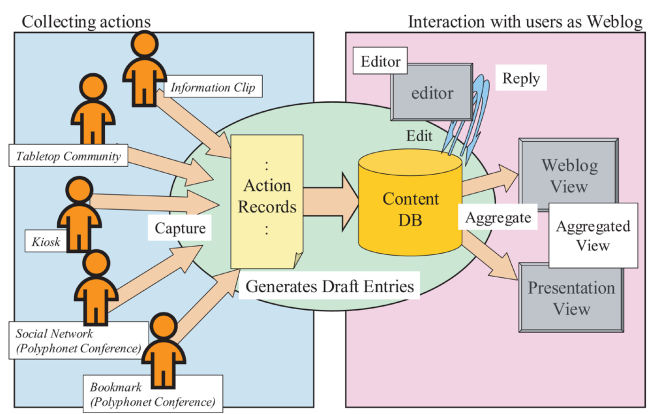

Figure 2: System architecture of the ActionLog system

\section{CAPTURING USERS' ACTIONS}

\subsection{Overall}

ActionLog-C collects the user's action from other UbiCoSup subsystems. UbiCoSup consists of three Web-based and three on-site subsystems as follows:

- Web-based subsystems:

- Researchers' social network system (part of Polyphonet Conference)

- Scheduling support system (part of Polyphonet Conference)

- Action-oriented Weblog system (ActionLog)

- On-site subsystems:

- Kiosk PC terminal using RFID card for user authentication 
- Photograph sharing and visualizing system based on an omni-directional camera and RFID card (Tabletop Community)

- Presentation annotating system based on mobile telephone (Information Clip)

These subsystems are built for the purpose of supporting participants in academic conferences. Researchers' information and their social relations are useful for understanding the research community and for contacting them. The scheduling support system helps participants to check presentations/demonstrations, by showing information about them i.e., authors, titles, and abstracts. Users can add a presentation to her schedule if she likes it. Participants can access the Web-based subsystems and view the social network using the kiosk PC settled in the conference site. Participants can communicate with others on the Tabletop Community, viewing their photographs and their networks. The Information Clip provides ways of checking presentations on site, annotating them with a mobile telephone. The user scans QR codes or RFID tags that are attached to panels set close to poster presentations and demonstrations using camera-enabled mobile telephones or mobile telephones with RFID tag readers, then the user can add comments on the presentations, and rate them.

User's action records on these subsystems are captured and integrated by ActionLog-C. We briefly introduce other UbiCoSup subsystems and define the actions captured from them.

\subsection{Polyphonet Conference}

The Polyphonet Conference is a social network browser and a conference scheduling system. Polyphonet obtains attendees' relationships and research topics from web sites. A user can find what research topic a particular researcher is doing or whom they are working with. In the scheduling part, a user can register interesting presentations (papers, demos, and posters) and get recommended presentations and other researchers.

Polyphonet has three methods to extract social networks among participants. The first one is based on Web mining techniques. A Web mining method is applied to extract a social network among the participants (Matsuo, 2003). Roughly speaking, our approach is to measure the relevance of two nodes based on the number of retrieved results obtained by a search engine query, e.g., "Mark Weiser and Alan Kay."

The second one is based on a user's operation on the Web system, and the third one is captured from users' interaction on the conference site.

Two types of actions are captured. One is to add a person to the acquaintance list (Action 1), and the other is to add a presentation to someone's own schedule (Action 2).

\subsection{Kiosk PC}

We place kiosk PCs at the conference site. Participants can access Web systems. We distribute RFID cards to participants. The kiosk uses RFID cards for user authentication. Each kiosk has three RFID card readers. A user can log into the Web system by putting her card on one of card readers. In addition, participants can see social networks among them when two or three participants put IC cards together. Then the social-tie, "We will meet and observe social networks together," is automatically added to Polyphonet Conference.

The kiosk PC sends a user's action, i.e., viewing the social network with other users (Action 3), to ActionLog.

\subsection{Tabletop Community}

The Tabletop Community is a system based on the idea of a micro-community similar to that created around tables and situations where people are drinking coffee in a casual way. Therefore, the environment that the system is targeting is something similar to corners of conference rooms and places for coffee breaks. We have developed the system shown in Figure 3. An omni-directional camera and RFID card readers are on the table of the system. Using these camera and card readers, when the user puts an RFID card onto a reader, the system detects the individual's rough location (direction of the user from the camera) and identification of users in the picture, which is taken by an omni-directional camera, every time a user places/withdraws a personal RFID card.

When two or three participants put IC cards together, they can see the network of their photographs shown in Figure 3.

The Tabletop Community recognizes the user's actions when she took photographs and interacted with other participants (Action 4).

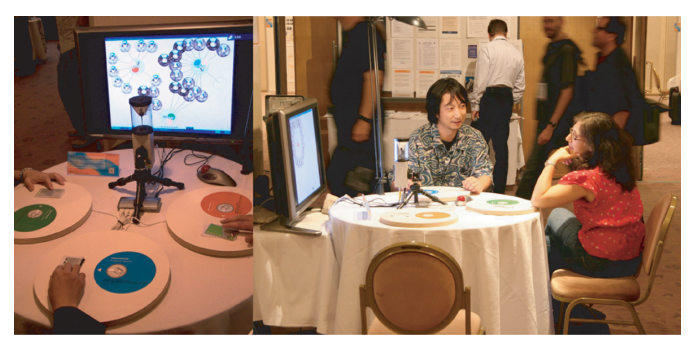

Figure 3: Tabletop Community usage 


\subsection{Information Clip}

Conferences have numerous presentations and demonstrations, but participants have difficulty recalling details and onsite impressions. We provide an environment for information clipping by applying mobile telephones (Figure 4). By scanning a clip signal, such as a QR code that is printed on the conference program or RFID tags on a poster, an application user can record an interesting demonstration and can save pictures or comments as an impression. In addition, users can share their pictures and comments with presenters.

In the Information Clip, two types of actions are identified, i.e., checking a presentation on a conference program (Action 5) and actually attending a presentation on site (Action 6).

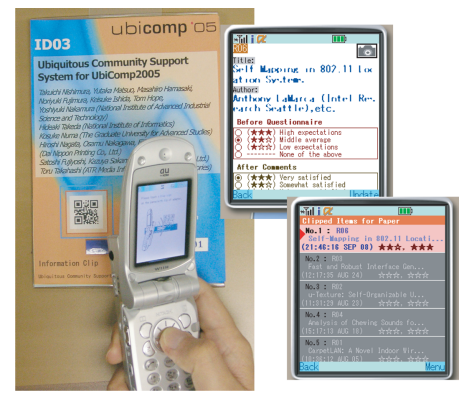

Figure 4: Information Clip usage

\subsection{Designing Users' Actions}

Through the subsections above, we described the subsystems of UbiCoSup. In the ActionLog system, the participant assistant agent captures users' actions from these systems. These six kinds of captured actions are categorized into two types: actions related to people and actions related to presentations. Actions related to people represent communication activities. Actions related to presentations encourage shared opinions about presentations. Table 1 categorizes the captured actions.

\section{WEBLOG CONTENT GENERATION AND REPRESENTATION}

Enhancing communications among participants is a key to achieving an active community. Actionoriented experience sharing promotes the exchange of opinions. In the system, user's actions are captured and collected from other information systems.
Based on the context information of the action, the system generates the Weblog content associated with the action. The user's action records are automatically generated through interactions with other systems and other people in the event space and on the Web. The user can edit the generated contents. They are published on the Weblog and are shared among participants. Exchanging contents among users can enhance communications. Aggregating the contents based on their context facilitates context-based access to the contents; responding to others' contents engenders topic-based discussion.

\subsection{Collecting Users' Actions, Generating and Publishing Weblog Contents}

Users' actions are sent by UbiCoSup subsystems for the ActionLog system via HTTP POST requests. A subsystem sends a request that includes queries, such as (UserID, Place, Actiontype, ContextInformation). ContextInformation depends on its Actiontype, which is pre-registered to ActionLog. The ActionLog system interprets the Contextinformation based on its ActionType, and then it generates the contents.

The ActionLog system generates a Weblog entry for each action sent from a UbiCoSup subsystem, interpreting the context information of the actions. Interpretation of the context information differs according to the types of actions. Take a Bookmark action for example. A bookmark action is sent from Polyphonet Conference and its context information is a presentation ID. The ActionLog agent generates a Weblog entry using the following steps.

1. Fetch the title and the authors' names associated to the Presentation ID.

2. Get a list of users who added the presentation as a bookmark from Polyphonet Conference (scheduler).

3. Get a list of user's acquaintances from Polyphonet Conference (social network).

4. Make an intersection of these two lists.

5. Apply the template and generate the content.

An example of the output is shown in Figure 5. These procedures, the content templates, and the type definition of the context information are also preregistered to the ActionLog system.

Generated contents are inserted to the user's Weblog. The context information, e.g. the presentation that was attended, the place this action occurred, and so on, is attached to the contents. A user can edit the 
Table 1: Types of captured actions

\begin{tabular}{|c|c|c|c|c|}
\hline No. & Action & System & Action Type & Context Information \\
\hline 1 & Adding a person to the acquaintance list & Polyphonet Conference & People & Participant ID \\
\hline 2 & Bookmarking(adding to schedule) a presentation & Polyphonet Conference & Presentation & $\begin{array}{l}\text { Presentation ID, } \\
\text { List of Participants who bookmarked the same presentation }\end{array}$ \\
\hline 3 & Viewing the social network among two or three users & Kiosk PC & People & Participant ID \\
\hline 4 & tTaking a photograph with other participants & Tabletop Community & People & Participant ID \\
\hline 5 & Checking a presentation on a conference program & Information Clip & Presentation & Presentation ID, User' s Comment \\
\hline 6 & Attending a presentation & Information Clip & Presentation & Presentation ID, User's Comment \\
\hline
\end{tabular}

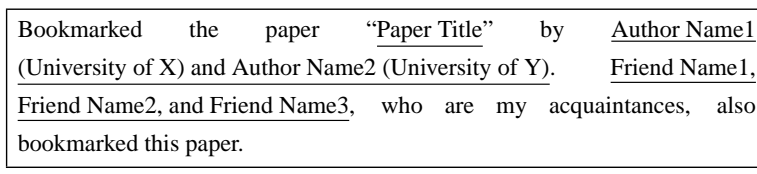

Figure 5: Example of generated content

contents on the editor page (Figure 6). She can remove or modify the generated sentences, or add new sentences.

Figure 7 shows a snapshot of the ActionLog system menu page. On the menu page, the captured actions, i.e., the generated contents, are listed in the red box. The user can edit and publish the generated contents. Figure 8 shows the Weblog-style view, which lists the user's published contents in chronological order.

\subsection{Aggregating and Sharing Contents}

Efficient sharing of published contents is achieved through content aggregation. We provide a contextbased aggregation view of contents.

Kaenampornpan et al. (Kaenampornpan, 2004) proposed the context model for context-aware systems based on Activity Theory (Engeström, 1999). According to the model, contexts are classified to six elements: User, Tools and their availability, Rules, Community, Division of Labour, Object, and Time.

In explanation of our proposed system, User is the user herself and the place she is. Tools represent each UbiCoSup subsystem. Rules are the norms and the social rules of the conference, and Community includes the people around the user. Division of Labour is the role in the action, such as the presenter or attendee. Object is the target of the action, such as the presentation that the user attended or the person that the user added to the acquaintance list. Time is the time at which the action occurred.

The Weblog-style view is a person-based view of the contents in chronological order. It includes the User, Community, and Time items. The Tools, Divi- sion of Labour and Object items are closely related to the type of action. We provide aggregation views on the types and targets of actions. We do not target Rules in this system.

The basic idea of aggregation is filtering according to context. The system extracts and lists contents related to the same presentation (Figure 9). This type of aggregation treats Object contexts. The system also aggregates by type of action. In this case, Tools and Division of Labour are considered. As we previously mentioned, the personal Weblog-style view includes User and Community based aggregation. The user can look through all the contents related to the context that the user specifies.

\subsection{Supporting Communications}

Only aggregation is insufficient to enhance communication among users. Sharing experiences is an important factor in understanding other people, but after understanding, we must interact. For this purpose, we provide the "reply" function. This function allows users to write new content that includes opinions, impressions, advice, and any other comment about another's content.

The user can create a replying content by clicking the "reply" buttons shown at the bottom of each section of the content. This function is implemented based on TrackBack. TrackBack is a typical Weblog feature that automatically creates reverse links among contents. The proposed system locates the reply contents using the following reverse links and presentations.

Furthermore, we provide an aggregated content list on the editor page (Figure 6). Users can edit their own actions while reviewing other contents that share context with the editing action. This capability promotes users' acquisition of new opinions and information about the action or the target of the action. 


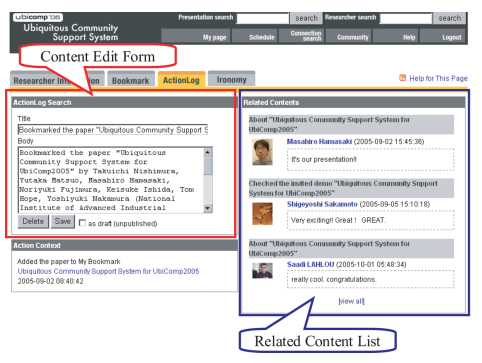

Figure 6: Editor page of ActionLog

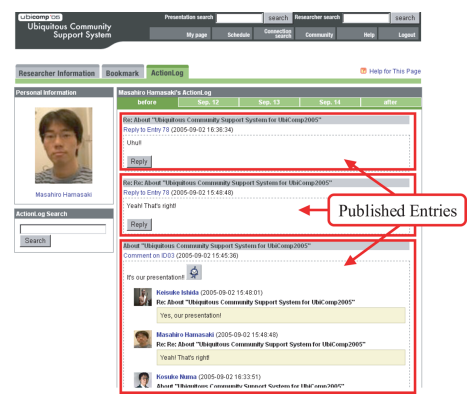

Figure 8: Weblog-style view of ActionLog

\section{RESULTS}

We tested our system at the 7th International Conference on Ubiquitous Computing (UbiComp2005), which was held on the 12th-14th of September 2005, in Tokyo. At this conference, there were 122 presentations and 355 authors (including co-authors). About 500 participants joined the conference.

There were 308 Polyphonet users, 175 users registered from the web system, and 133 users were invited from information kiosks in the conference room.

Regarding ActionLog, 4586 entries were generated. There were 3918 entry contents from the actions related to people and 668 entries for presentations contents.

Finally, 105 entries were published. Fifteen entries were generated from actions related to people, 64 entries from presentations, and 26 entries were not generated from actions, i.e., interactions with others, which included replying entries.

Figure 10 shows the rates of the entries for actions. Contents related to presentations increased in rate when published. The rate of replying contents also occupied a large part. These facts show that a topic-centered view played an important role in sharing contents among users. Actually, several discussions were made in the ActionLog system.

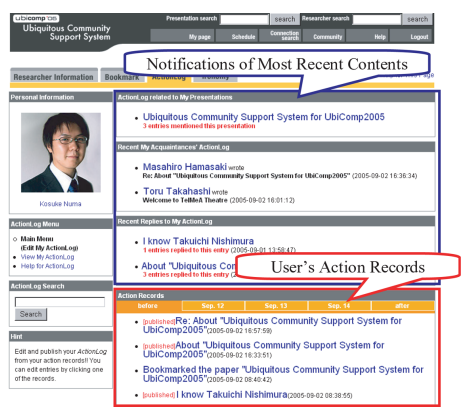

Figure 7: Menu page of ActionLog

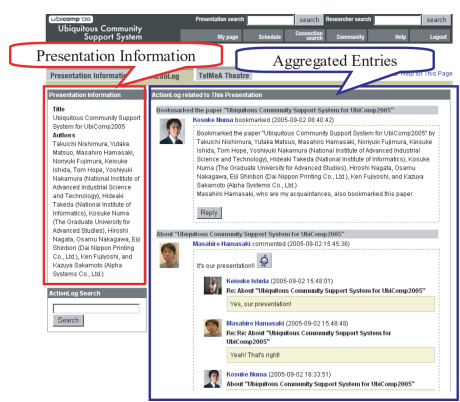

Figure 9: Presentation-based aggregated view of ActionLog

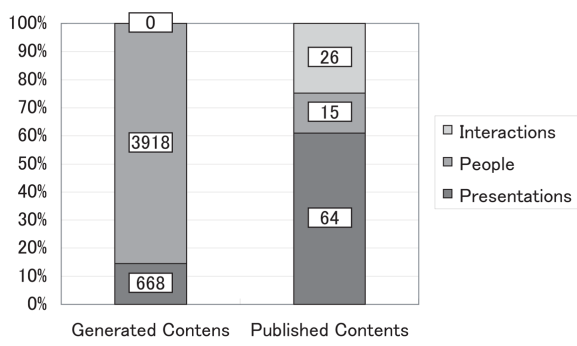

Figure 10: Rates of entries for actions

\section{RELATED WORK}

Several conference support systems have been proposed. IntelliBadge (Cox, 2003) is an RFID-based academic conference support system that traces users' positions and provides location search services for users. It enhances communication at conference sites, but does not support post-conference communication.

Another system, C-MAP (Sumi, 1998), is a personal mobile assistance system for exhibition tours. It is intended as a location-aware support by sharing experiences and knowledge using mobile terminals like PDAs. It provides total support in an event space, but does not target integrative support of independent subsystems in the real world or on the Web. 
LifeLog research, like MyLifeBits (Gemmell, 2002), is a salient approach to sharing experiences by recording everything users see and hear. However, our approach is to share users' subjective experiences rather than objective records.

SPECTER (Kröner, 2004) is a personal journal generation system that generates content based on the users' action records. This approach closely resembles our approach, but it is specifically intended to model users' behaviors and does not target supporting communications or sharing experiences.

\section{CONCLUSION}

We propose a Weblog with a real-world context called ActionLog. ActionLog gathers users' actions, with related context, and generates entry drafts indeed of users. With ActionLog, users can accumulate what they accomplish in small increments as draft entries, and publish these entries, after they edit generated drafts. The published entries can be easily aggregated with various aspects because they have context as metadata.

ActionLog applies to conference support. The ActionLog Conference worked as an integrator of other conference support systems. It collects and seamlessly integrates users' actions from the Web and the real world. The collected actions are shown in various styles according to their state of use.

The action-oriented integration is easily extensible. New subsystems are pluggable through "action." This means that our approach can be applied in various situations. Future work includes designing a system with more proactive supports. The tested implementation is a limited service in terms of time and place. When we utilize the system in an open environment, the information that will become available will be enormous so that a user cannot manage them.

\section{ACKNOWLEDGEMENTS}

The Polyphonet Conference has been supported by the New Energy and Industrial Technology Development Organization (NEDO) as the 04A11502a project ID. The cellular phones with RFID tag readers for the conference were courtesy of KDDI Corporation. The IC card reader system and technology are supported by Synergymedia Corporation.

\section{REFERENCES}

Avesani, P., Cova, M., Hayes, C., and Massa, P. (2005). Learning Contextualised Weblog Topics. In Proc. of WWW2005 2nd WS on the Weblogging Ecosystem: Aggregation, Analysis and Dynamics.

Cox, D., Kindratenko, V., and Pointer, D. (2003). IntelliBadge: Towards Providing Location-Aware ValueAdded Services at Academic Conferences. In Proc. of 5th Int'l Conf. on Ubiquitous Computing (UbiComp2003).

Duckham, M. and Kulik, L. (2005). A formal Model of Obfuscation and Negotiation for Location Privacy. In Proc. of 3rd Int'l Conf. on Pervasive Computing (Pervasive2005).

Engeström, Y. (1999). Activity theory and individual and social transformation. In Y. Engestrom, R. Miettinen and R. Punamaki (eds.), Perspectives on Activity Theory, Cambridge University Press.

Gemmell, J., Bell, G., Lueder, R., Drucker, S., and Wong, C. (2002). MyLifeBits: fulfilling the Memex vision. In Proc. of 10th ACM Int'l Conf. on Multimedia.

Gruteser, M. and Grunwald, D. (2003). Anonymous usage of location-based services through spatial and temporal cloaking. In Proc. of 1st Int'l Conf. on Mobile Systems, Applications and Services (MobiSys'03).

Kaenampornpan, M. and O’Neill, E. (2004). Modelling Context: An Activity Theory Approach. In Proc. of 2nd European Symposium on Ambient Intelligence (EUSAI2004).

Karger, D. and Quan, D. (2004). What Would It Mean to Blog on the Semantic Web? In Proc. of 3rd Int'l Semantic Web Conf. (ISWC2004).

Kröner, A., Baldes, S., Jameson, A., and Bauer, M. (2004). Using an Extended Episodic Memory Within a Mobile Companion. In Proc. of Pervasive 2004 WS on Memory and Sharing of Experience (MSE2004).

Matsuo, Y., Tomobe, H., Hasida, K., and Ishizuka, M. (2003). Mining Social Network of Conference Participants from the Web. In Proc. of 2003 IEEE/WIC Int'l Conf. on Web Intelligence (WI2003).

Newman, W., Eldridge, M., and Lamming, M. (1991). PEPYS: Generating Autobiographies by Automatic Tracking. In Proc. of 2 nd European Conf. on Computer-Supported Cooperative-Work (ECSCW'91).

Sumi, Y., Etani, T., Fels, S., Simonet, N., Kobayashi, K., and Mase, K. (1998). C-MAP: Building a ContextAware Mobile Assistant for Exhibition Tours. In Toru Ishida (Ed.), Community Computing and Support Systems: Social Interaction in Networked Communities, LNCS 1519, Springer.

Sumi, Y., Sakamoto, R., Nakao, K., and Mase, K. (2002). ComicDiary: Representing Individual Experiences in a Comics Style. In Proc. of 4th Int'l Conf. on Ubiquitous Computing (UbiComp2002). 